\title{
THE "MINUTE" CYTOPLASMIC VARIANT OF ASPERGILLUS NIDULANS
}

\author{
B. M. FAULKNER* and C. F. ARLETT†
}

Department of Genetics, University of Birmingham

Received 14.vii.63

\section{INTRODUCTION}

THE recovery of cytoplasmic variants from fungi, following treatment with acriflavine, has been reported for Saccharomyces cerevisiae (Ephrussi, Hottinguer and Chimènes, 1949; Ephrussi, 1953; Ephrussi, Hottinguer and Roman, 1955); Neurospora crassa (Srb, 1958, I963); and Aspergillus nidulans (Arlett, I957; Roper, 1958).

The present paper describes some of the properties of a variant obtained by Arlett (1957) after treatment of a wild type strain of $A$. nidulans with acriflavine.

Unless otherwise stated, colonies were grown on Czapek's minimal medium at $25^{\circ} \mathrm{C}$.

\section{INDUCTION OF THE MINUTE VARIANT}

The minimum concentration of acriflavine in minimal medium required for a 100 per cent. kill of conidia from the wild type strain of A. nidulans, was found to be $\mathrm{I}$ in $7.5 \times 10^{4}$ parts by weight. Mass inoculations of wildtype hyphæ onto this concentration of acriflavine grew very slowly. The inocula in 28 days of growth produced only very small colonies. Samples of conidia from two such small colonies were plated on minimal medium, and on media containing acriflavine at concentrations ranging from $I$ in $10^{5}$ to $I$ in $10^{4}$ parts by weight. None of the conidia on the acriflavine supplemented media germinated. The conidia on minimal medium produced 289 apparently normal colonies and 5 colonies with the minute phenotype. Four of the minute colonies came from one of the small parent colonies. Three facts suggested that these minute colonies were induced rather than selected by the acriflavine treatment. Minute colonies exhibited no greater tolerance of acriflavine than did wild type colonies and phenotypically indistinguishable minute colonies were recovered from two independently treated parent colonies. Furthermore, the wild type strain never spontaneously produced minute colonies.

\section{THE NATURE OF THE MINUTE VARIANT}

The variant colonies were called minute because they were smaller than normal colonies. The minute colonies produced fewer conidial

* Present address: Department of Botany, Bedford College (University of London), Regent's Park, N.W.1.

$\dagger$ Present address: Wantage Research Laboratory (A.E.R.E.), Wantage, Berks. 
heads and perithecia, than did the normal colonies. The perithecia of minute occurred in clumps, and the conidial heads were more frequently produced by the Hülle-cell hyphæ, which surround the perithecia (plate II $b$ ), than by hyphæ elsewhere in the mycelium. Minute colonies produced very little of the reddish-brown pigment characteristic of the wild type strain.

\section{(i) Persistent segregation}

Culturing of minute revealed that it was a mosaic. Asexual spores from minute gave rise to both minute and apparently normal colonies (plate $\mathrm{I} a$ ). The latter we shall call $m$-normal. The $m$-normal segregants grew, on average, 80 per cent. faster than the minute segregants. The segregation was repeated by the asexual spores taken from the minute segregants. Analysis of the progenies from individual conidial

TABLE I

Two examples of the sexual spore progeny produced by the minute variant

\begin{tabular}{|c|c|c|c|}
\hline \multirow{2}{*}{$\begin{array}{c}\text { Minute } \\
\text { colony }\end{array}$} & $\begin{array}{c}\text { Number of } \\
\text { perithecia }\end{array}$ & $\begin{array}{c}\text { Phenotype of sexual } \\
\text { spore progeny }\end{array}$ \\
\cline { 3 - 4 } & & $m$-normal & minute \\
\hline I & 5 & 7965 & 0 \\
2 & 5 & 8537 & 0 \\
\hline
\end{tabular}

heads showed that the segregation was also occurring among conidiophores. Some conidiophores gave rise to both $m$-normal and minute offspring, whereas others gave rise to only $m$-normal offspring.

Minute colonies produced sectors which were identical in growth rate and morphology with $m$-normal colonies, and which, like $m$ normal, gave only $m$-normal asexual and sexual progeny. These sectors we shall call $m$-normal sectors (plates $I a$ and $I b$ ). The first sign of the production of a $m$-normal sector was the growth of hyphæ from the margin of a minute colony at a point where a clump of perithecia abutted on it (plate II $a$ ). This sectoring of minute was shown to be due to segregation of the minute and $m$-normal phenotypes amongst single hyphæ. Subculturing of minute by means of single hyphæ, taken from the growing front, gave $m$-normal and minute colonies. The latter, in their turn, segregated in the production of $m$-normal sectors (plate Ic).

The segregation minute: $m$-normal has been repeated in every asexual progeny from minute colonies over a period of 6 years. A pure-breeding minute colony has never been found. In contrast, the sexual offspring of minute colonies have never shown this segregation. Minute colonies have never been obtained from amongst sexual spore progenies (table I). 


\section{(ii) Origin}

A heterokaryon test (Jinks, I954, I958), to distinguish between nuclear and extranuclear origin of a phenotypic difference, was found to be impracticable in the case of minute, because rapid breakdown of the minute mycelium to $m$-normal precluded the formation of heterokaryons involving minute.

A. nidulans has been reported as having uninucleate conidia (Yuill, I950). The conidia of the parent wild type strain proved on examination to be uninucleate. Therefore any attempt to ascribe minute to gene mutation must explain the persistence of the segregation, minute: $m$-normal, through uninucleate conidia. Such a segregation might occur through either haploidisation, or mitotic recombination, if minute possessed diploid nuclei heterozygous at a "minute" locus and if the mutant allele was dominant to the normal allele. In the case of haploidisation both diploid and haploid conidia should be produced by minute colonies; while persistent mitotic recombination in heterozygous, diploid nuclei would depend on the production of diploid conidia. The size difference recorded for haploid and diploid conidia of $A$. nidulans (Yuill, I950; Pontecorvo, I953) made possible a simple test of these two possibilities. Conidia from minute did not fall into two size-classes. Also conidia from both minute and $m$-normal colonies had mean diameters of 3.7 microns. Although these mean diameters were higher than those previously reported for haploid conidia of $A$. nidulans, they fell below those reported for diploid conidia. The nuclei of minute, and $m$-normal, conidia were evidently haploid. Accordingly gene mutation and diploidisation were rejected as unlikely explanations of the origin of minute.

The coincidence of persistent segregation and a uninucleate, haploid condition of the asexual spores has been taken as presumptive evidence of an extranuclear origin of the segregation (Jinks, I963). Such an interpretation has been substantiated, in the case of the ultraviolet induced, persistently segregating, "red " variant of $A$. nidulans (Arlett, Grindle and Jinks, I962), by means of heterokaryon tests. The minute variant was derived from the same wildtype strain of A. nidulans as the red variant. Although the patterns of persistent segregation are not identical in the two variants, we suggest that they are similar in origin, and that minute originated from a change in the cytoplasm of the wildtype strain.

The supposed cytoplasmic change, responsible for the appearance of the minute phenotype, could have occurred either through loss or by the change in function of a cytoplasmic determinant. A choice between these alternatives can be made with respect to the occurrence of persistent segregation. Persistent cytoplasmic segregation is possible only where alternative states exist together within the same cytoplasm. If minute resulted from the loss of a cytoplasmic determinant, there would appear to be no basis for the segregation minute: $m$-normal. In 
the case of a change in function, segregation is explicable in terms of the distribution, amongst hyphæ, conidiophores and conidia, of cytoplasmic determinants of normal and changed function. We conclude that parts, at least, of the mycelium of minute are heteroplasmic (Jinks, I96I), functionally normal determinants being present alongside functionally mutant determinants in the minute cytoplasm.

\section{(iii) The heteroplasmon}

The proposed minute heteroplasmon is theoretically capable of giving rise to three classes of asexual offspring. These are: purebreeding, homoplasmic $m$-normal; segregating, heteroplasmic minute; and pure-breeding, homoplasmic minute. Examples of the latter class have never been recovered. Therefore, in espousing the heteroplasmon model, we must conclude that a cytoplasm containing only the mutant form of the determinant is either lethal or subject to the backmutation of the mutant to the normal determinant, at such a rate as to make homoplasmic minute undetectable. There are no firm pointers to either of these explanations. However, amongst the progeny of single, heteroplasmic conidiophores, $m$-normal colonies were observed to be more frequent than minute colonies in 98 per cent. of cases. This may reflect an absence, through lethality, of homoplasmic minute progeny. Similar difficulties in distinguishing between lethality and backmutation as the mechanism responsible for the absence of a mutant homoplasmon have been experienced in connection with the red cytoplasmic variant of $A$. nidulans (Arlett, Grindle and Jinks, 1962). In this case, where more evidence was available than for minute, lethality was chosen as the most likely mechanism.

Because all its sexual progeny and the majority of its asexual progeny are homoplasmic the minute heteroplasmon is unstable. Indeed, it has only proved possible to maintain the heteroplasmon by continuous selection of minute colonies from among the asexual progenies of parent minute colonies. The normal cytoplasmic determinant has a greater chance than the mutant determinant of inclusion in a conidium or a hypha, and enjoys a total monopoly in this respect in the case of sexual spores.

\section{(iv) Suppression}

The normal and mutant cytoplasmic components of the minute heteroplasmon are both suppressive in the senses defined in previous studies of fungi (Ephrussi, Hottinguer and Roman, I955; Jinks, 1959, 196I). The expression of the minute phenotype by the heteroplasmon involves the "suppression" by the mutant cytoplasmic determinants of the activity of the normal determinants. The breakdown of the heteroplasmon in the production of pure-breeding, $m$-normal homoplasmons involves the "suppression" of the mutant determinants by the normal determinants during growth and reproduction. This second type of suppressive activity is related to the distribution of the heteroplasmon in the cytoplasm of the minute colony. 


\section{DISTRIBUTION OF THE HETEROPLASMON IN THE MINUTE MYCELIUM}

The occurrence, in the mycelium of a minute colony, of perithecia and conidiophores which gave rise to only $m$-normal progeny, and of single hyphæ which grew into $m$-normal colonies, suggested that not all of the minute mycelium was heteroplasmic. Rather it was a mosaic made up of minute heteroplasmon and $m$-normal homoplasmon. The distribution of heteroplasmon and homoplasmon in the minute mycelium was revealed by studying the distributions of conidiophores, perithecia and hyphæ within the mycelium, and the progenies to which they gave rise.

\section{(i) Conidiophores}

The process of asexual spore formation involves the isolation of a small sample of cytoplasm from the much larger mass present in the conidiophore itself. Thus when a conidiophore gives rise to both $m$-normal and minute progeny, the conidiophore is itself clearly heteroplasmic, in that it produces both heteroplasmic and homoplasmic conidia. When, however, a conidiophore produces only $m$-normal progeny, the nature of its cytoplasm cannot be so confidently asserted. Nevertheless, in the absence of any indication of competition between normal and mutant determinants for inclusion in the cytoplasm of the conidia and of a threshold effect associated with the suppressive activity of the mutant cytoplasmic determinant, we consider it unlikely that a heteroplasmic conidiophore, even if it contained but few representatives of the mutant determinant, would not produce some minute progeny. We therefore interpret the absence of minute progeny as meaning the absence of the mutant determinant from the cytoplasm of the conidiophore.

Two discrete sites of conidiophore production were recognised within the minute mycelium. They were "perithecial" sites, where the conidiophore was situated on a perithecium among the Hülle cells (plate II $b$ ), and "non-perithecial " sites where the conidiophore was situated directly on the hyphal mass of the mycelium.

The progenies of 97 conidiophores, taken from a single minute colony, were separately analysed. The conidiophores were taken at random, 54 from perithecial sites, and 45 from non-perithecial sites, and were subsequently classified as homoplasmic or heteroplasmic according to progeny phenotypes. The results are summarised in table 2. Heteroplasmic and homoplasmic conidiophores were equally frequent in the minute mycelium, but they were not evenly distributed between perithecial and non-perithecial sites. A contingency $\chi^{2}$ test $\left(\chi_{(1)}^{2}=36.5, \mathrm{P}<0.00 \mathrm{I}\right)$ showed that there were significantly more heteroplasmic conidiophores than homoplasmic conidiophores at non-perithecial sites; while the converse was true of perithecial sites. Minute colonies constituted a larger percentage of the total asexual progeny from the 43 non-perithecial sites, than of the total asexual 
progeny from the 54 perithecial sites. This latter observation was confirmed by a comparison of similar sized samples of progeny from 20 conidiophores at perithecial, and 20 conidiophores at non-perithecial,

TABLE 2

Distribution of homoplasmons and heteroplasmons among 97 conidiophores from perithecial, and non-perithecial, sites of a minute colony

\begin{tabular}{|c|c|c|c|}
\hline \multirow{2}{*}{$\begin{array}{l}\text { Site in minute } \\
\text { mycelium }\end{array}$} & \multicolumn{3}{|c|}{$\begin{array}{c}\text { Conidiophores classified on } \\
\text { progeny phenotypes }\end{array}$} \\
\hline & Homoplasmic & Heteroplasmic & Total \\
\hline $\begin{array}{l}\text { Perithecial } \\
\text { Non-perithecial }\end{array}$ & $\begin{array}{r}42 \\
6\end{array}$ & $\begin{array}{l}12 \\
37\end{array}$ & $\begin{array}{l}54 \\
43\end{array}$ \\
\hline Total . & $4^{8}$ & 49 & 97 \\
\hline
\end{tabular}

sites of four minute colonies (table 3 ). Two factors probably contributed to this result. As table 2 shows, perithecial sites in the minute mycelium give rise less frequently to heteroplasmic conidiophores than do non-perithecial sites. Furthermore, minute colonies constituted

TABLE 3

Asexual spore progenies from perithecial, and non-perithecial, sites of four minute colonies

\begin{tabular}{|c|c|c|c|c|}
\hline \multirow{2}{*}{ Colony } & \multirow{2}{*}{ Site } & \multicolumn{3}{|c|}{ Asexual progeny } \\
\hline & & $m$-normal & minute & $\begin{array}{c}\text { percentage } \\
\text { minute }\end{array}$ \\
\hline I & $\begin{array}{l}\text { perithecial } \\
\text { non-perithecial }\end{array}$ & $\begin{array}{l}\text { I } 59 \text { I } \\
\text { I } 300\end{array}$ & $\begin{array}{l}136 \\
307\end{array}$ & $\begin{array}{r}8 \\
19\end{array}$ \\
\hline 2 & $\begin{array}{l}\text { perithecial } \\
\text { non-perithecial }\end{array}$ & $\begin{array}{r}996 \\
1055\end{array}$ & $\begin{array}{l}167 \\
300\end{array}$ & $\begin{array}{l}14 \\
22\end{array}$ \\
\hline 3 & $\begin{array}{l}\text { perithecial } \\
\text { non-perithecial }\end{array}$ & $\begin{array}{r}\text { I } 372 \\
784\end{array}$ & $\begin{array}{r}29 \\
323\end{array}$ & $\begin{array}{r}2 \\
29\end{array}$ \\
\hline 4 & $\begin{array}{l}\text { perithecial } \\
\text { non-perithecial }\end{array}$ & $\begin{array}{r}\text { 1 } 476 \\
98 \text { I }\end{array}$ & $\begin{array}{r}45 \\
363\end{array}$ & $\begin{array}{r}3 \\
27\end{array}$ \\
\hline
\end{tabular}

8 per cent. of the total progeny from the 37 heteroplasmic conidiophores at non-perithecial sites, but only 2 per cent. of the total progeny from the I2 heteroplasmic conidiophores at perithecial sites. It would appear, therefore, that heteroplasmic conidiophores at perithecial sites produce heteroplasmic conidia less frequently than do heteroplasmic conidiophores at non-perithecial sites. 
The frequency of occurrence of heteroplasmic conidia can be used as a measure of the frequency of mutant cytoplasmic determinants within a heteroplasmic conidiophore, if it is assumed that normal and mutant determinants are distributed at random within the conidiophore cytoplasm. Thus, not only is the cytoplasm of conidiophores at perithecial sites less likely to contain any mutant determinants at all, but where it does, the mutant determinant is possibly less frequent than in the cytoplasm of heteroplasmic conidiophores at non-perithecial sites.

\section{(ii) Perithecia and hyphæ}

The ascospores, from perithecia situated on the minute mycelium, give rise to pure-breeding $m$-normal colonies only. These ascospores therefore contain the normal, but not the mutant, cytoplasmic determinant. It is not equally certain, however, that all other parts of these perithecia contain only normal cytoplasm. For this reason it cannot be inferred, from the nature of the ascospores alone, that the hyphæ of the minute mycelium, which ultimately give rise to the ascospores, themselves contain only normal cytoplasm. The occurrence of perithecia on the margins of minute colonies at sites of origin of $m$-normal sectors (plate $\mathrm{II} a$ ) does, however, suggest that the perithecia are in some way associated with homoplasmic, $m$-normal hyphæ. The association was further investigated as follows. Seven-day-old minute colonies showed well defined $m$-normal sectors without sector overgrowth of non-sectoring parts of the minute mycelium. The sectors were made to delimit segments of the mycelium based upon the geometric centre of each colony. It was found that perithecia were not distributed at random over the minute mycelium, but were 88 per cent. more frequent per unit angle of segment in the segments of the mycelium bounded by $m$-normal sectors.

The production of $m$-normal sectors is evidence of the breakdown of the heteroplasmon in the minute mycelium. Some of the hyphæ in the minute mycelium must, therefore, contain only normal cytoplasm. Such $m$-normal hyphæ are the precursors of the $m$-normal sectors subsequently produced by the minute mycelium. It follows, therefore, that perithecia are most frequent in those segments of the minute mycelium where homoplasmic, $m$-normal hyphæ evidently occur.

We can further associate the perithecia with $m$-normal hyphæ by referring to the evidence from conidiophores. A conidiophore arises as a side branch of a mycelial hypha. The cytoplasm of the conidiophore should therefore reflect the condition of the cytoplasm of the hypha which produced it. Thus a homoplasmic, $m$-normal conidiophore is most likely to have originated from a homoplasmic, $m$-normal hypha. The predominance of homoplasmic, $m$-normal conidiophores at perithecial sites then indicates that the hyphæ, occurring in close proximity to perithecia within the minute mycelium, will be predominantly $m$-normal. 
Mass-hyphal transfers of minute mycelium onto fresh medium gave only $m$-normal colonies although, as previously mentioned, singlehyphal transfers produced either minute or $m$-normal colonies. The faster growth rate of $m$-normal hyphæ gives them a selective advantage over minute hyphæ in colonising the medium surrounding the masshyphal inoculum, such that minute asexual progeny were never found. It is possible, therefore, that no minute hyphæ grow out of the masshyphal inoculum. Comparison was made of the initial production of hyphæ by mass-hyphal inocula from perithecial, and non-perithecial sites of the minute mycelium, using inocula of a uniform size. The inocula were so orientated that only their hyphæ were in contact with the fresh medium, Perithecial minute mycelium produced more hyphae than did non-perithecial mycelium. It is possible that this result is yet further evidence of the greater frequency of homoplasmic, $m$-normal hyphæ in those parts of the minute mycelium where perithecia are situated.

The evidence from perithecia, homoplasmic conidiophores and $m$-normal sectors is in essential agreement regarding the distribution of the $m$-normal homoplasmon within the minute mycelium. Conversely, the distribution of heteroplasmic conidiophores within the minute mycelium indicates that the heteroplasmon occurs most frequently in those hyphæ which are not closely associated with perithecia.

\section{DISCUSSION}

The interpretation which has been put upon the results obtained concerning the minute variant of $A$. nidulans can be summarised as follows. The segregation of the minute: $m$-normal phenotypic difference among single conidia and hyphæ of the minute mycelium is brought about by the segregation of normal and mutant representatives of a particulate, hereditary determinant of the cytoplasm. The persistence of the segregation among single conidia and single hyphæ, themselves derived from single conidia and single hyphæ, means that these conidia and hyphæ can regularly contain both normal and mutant representatives of the determinant, i.e. these conidia and hyphæ are heteroplasmic. Persistence of the minute phenotype indicates a corresponding persistence of its mutant determinant, and persistence of the phenotypic change during asexual reproduction similarly indicates multiplication of that mutant determinant along with the normal determinant.

This interpretation provides the basis for an explanation of the behaviour and properties of the minute variant. Germination on solid medium of a heteroplasmic conidium produces an ever-increasing number of branching hyphæ which spread out over the medium in a radial fashion. Each hypha will contain representatives of the particulate hereditary determinant. The continual branching will provide many, and repeated, opportunities for the distribution of the normal and mutant determinants between hyphæ. With unequal 
distribution of determinants, some hyphæ will receive only normal determinants. Thus very soon after its initiation, the minute colony will be a mosaic of heteroplasmic minute, and homoplasmic $m$-normal, hyphæ. Subsequently this breakdown of the minute heteroplasmon is reflected in the growth and reproductive activity of the two kinds of hyphæ. Homoplasmic $m$-normal hyphæ give rise to $m$-normal hyphæ, sectors, conidiophores, conidia and ascospores. Similarly heteroplasmic minute hyphæ give rise to $m$-normal and minute hyphæ, conidiophores and conidia; but not to minute ascospores. Also it is doubtful whether minute hyphæ give rise to $m$-normal ascospores.

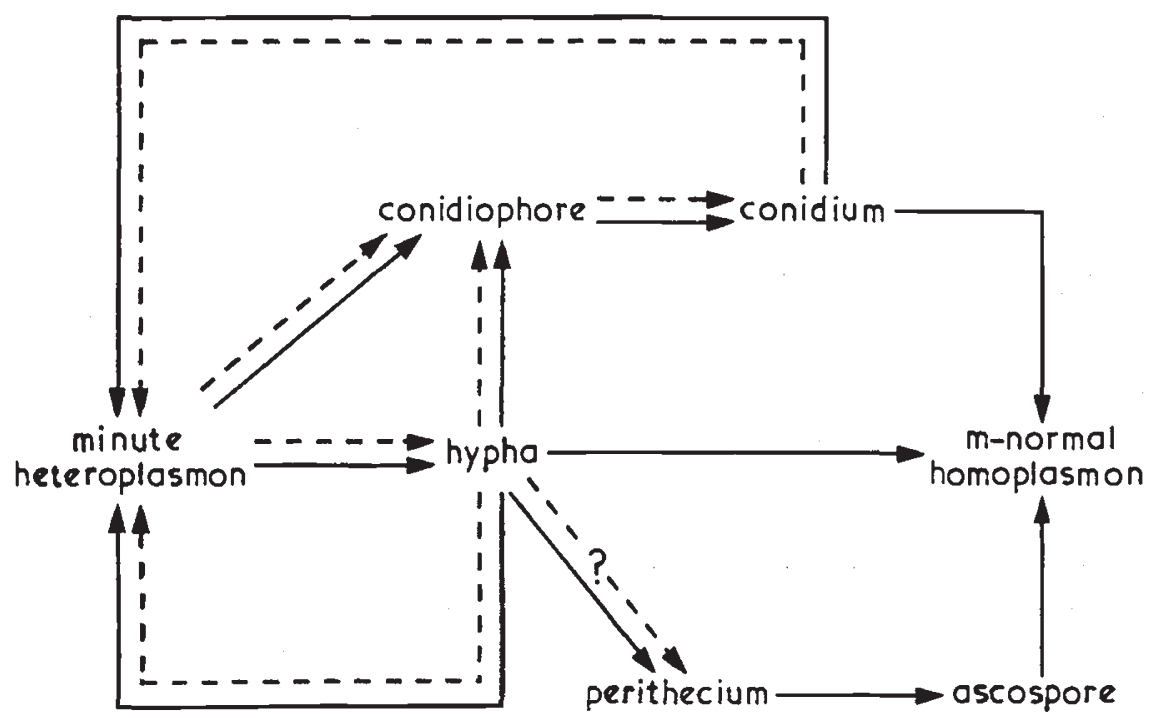

Fro. 1.-Transmission of the normal (-) and mutant (-.-) components of the minute heteroplasmon during growth and reproduction of the minute variant. The inclusion or non-inclusion of the mutant component in the pre-ascosporic cells of the perithecium is still uncertain.

The association between perithecia and $m$-normal hyphæ, together with the fact that all ascospores of minute are $m$-normal, suggests that $m$-normal hyphæ are more likely to give rise to ascospores. But this requires further analysis.

We can represent the life cycle of the minute variant as a continual recycling of the normal and mutant components of the cytoplasm through hyphæ, conidiophores and conidia; with diversion of the normal component alone into some hyphæ, conidiophores and conidia, and into all ascospores, resulting in the production of the $m$-normal homoplasmon (fig. I). Both the normal and mutant components of the cytoplasm survive asexual reproduction, but only the normal component survives sexual reproduction. It would appear that selection amongst pre-existing cytoplasmic variation is occurring in the differentiation of the sexual apparatus of the minute variant. The hyphæ- and conidia-forming potentialities of the wildtype nucleus of 
minute can be expressed in both homoplasmic normal, and heteroplasmic normal/mutant, cytoplasm. The ascospore-forming potentialities of the wildtype nucleus, on the other hand, can only be expressed in homoplasmic, normal cytoplasm. Finally, association of the wildtype nucleus with homoplasmic, minute cytoplasm is apparently lethal.

We have previously mentioned the occurrence of suppression in the minute cytoplasm. It can now be seen that suppression is not necessarily the direct outcome of an interaction between different cytoplasmic components. It may be, as is suggested by the present case, the expression at the phenotypic level of interactions between a nucleus and the different cytoplasms with which it is associated. Thus the suppressive activity of the mutant and normal components of the minute cytoplasm are both manifestations of differentiation and development in an induced, heteroplasmic mycelium. The mutant determinant suppresses the normal because the wildtype nucleus cannot behave normally in the heteroplasmon. Similarly the suppression of the mutant determinant by the normal resulting in the breakdown of the heteroplasmon, is a reflection of the superior activity of the nucleus in the normal homoplasmon. This breakdown of the heteroplasmon is made inevitable by the selective advantage in growth rate and reproductive capacity which the homoplasmon possesses.

The behaviour of the minute heteroplasmon, and the effect which it and its breakdown has on the growth and reproduction of the minute variant, leads us to conclude that the wildtype cytoplasm contains a particulate, hereditary component which plays a critical role in the determination of the normal pattern of differentiation and development of the wild type strain of $A$. nidulans.

\section{SUMMARY}

I. Acriflavine treatment of a wildtype strain of $A$. nidulans resulted in the recovery of the vegetatively unstable, minute variant.

2. The persistent segregation, minute: $m$-normal, in its asexual progenies suggests that the minute variant is a heteroplasmon between mutant and normal cytoplasmic determinants. Non-recovery of a pure-breeding, minute variant further suggests that the mutant cytoplasmic component is lethal in the homoplasmic state.

3. The continual breakdown of the minute heteroplasmon, during growth and reproduction, to give $m$-normal homoplasmons, results from the faster growth rate and reproductive capacity associated with the $m$-normal homoplasmon.

4. The distribution of perithecia over the minute mycelium is correlated with the distribution of the $m$-normal homoplasmon within the mycelium. It is therefore suggested that the $m$-normal homoplasmon, within the minute mycelium, is more likely than the minute heteroplasmon to bring about the production of the sexual apparatus of minute. 


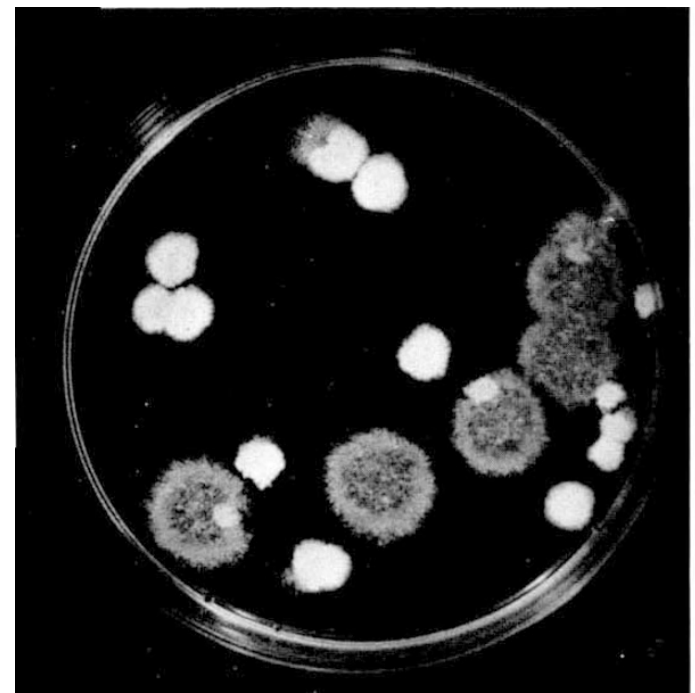

a.

The asexual progeny of a minute colony showing the segregation into two classes $m$-normal and minute. Several of the minute colonies are producing $m$-normal sectors.

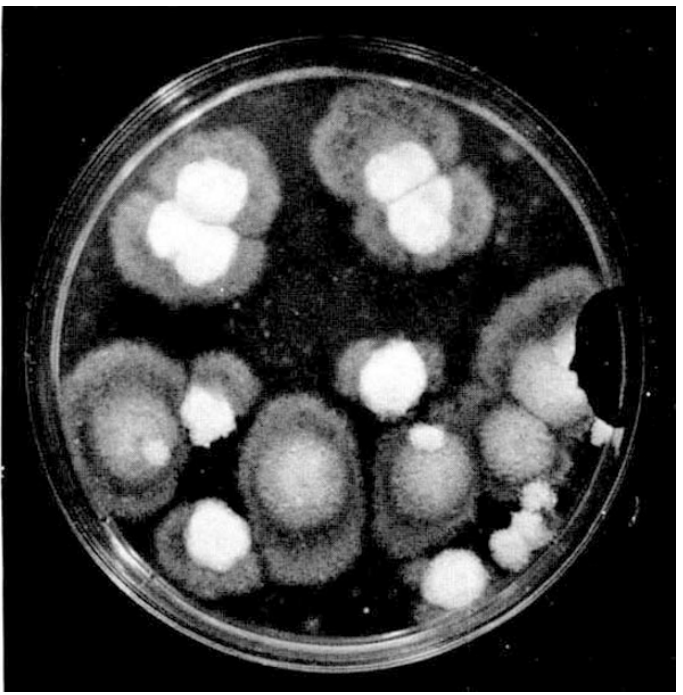

$b$.

Plate I $a$ three days later. Nearly all the minute colonies have produced one or more clearly discernable $m$-normal sectors.

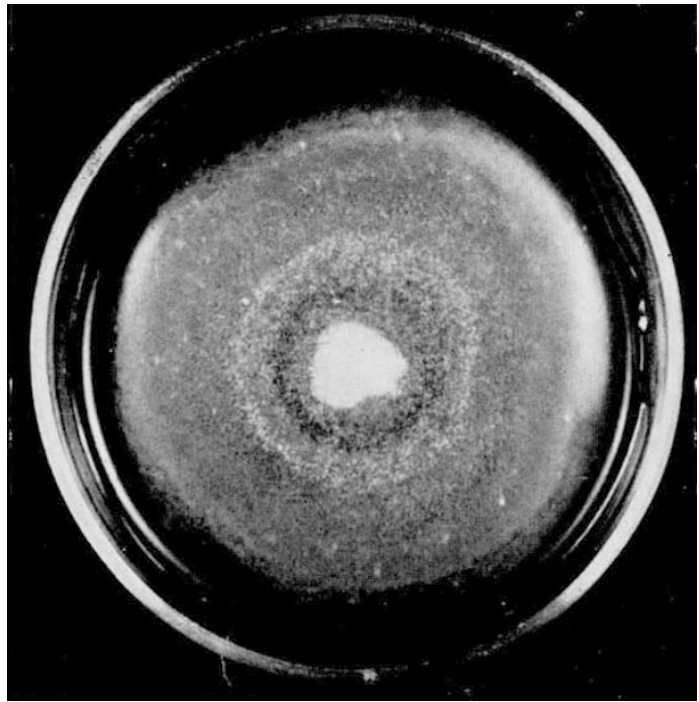

$c$.

A minute colony produced by a single hypha taken from the growing front of the parent minute colony. The minute mycelium has produced $m$-normal sectors which have coalesced and completely covered the surface of the medium. 


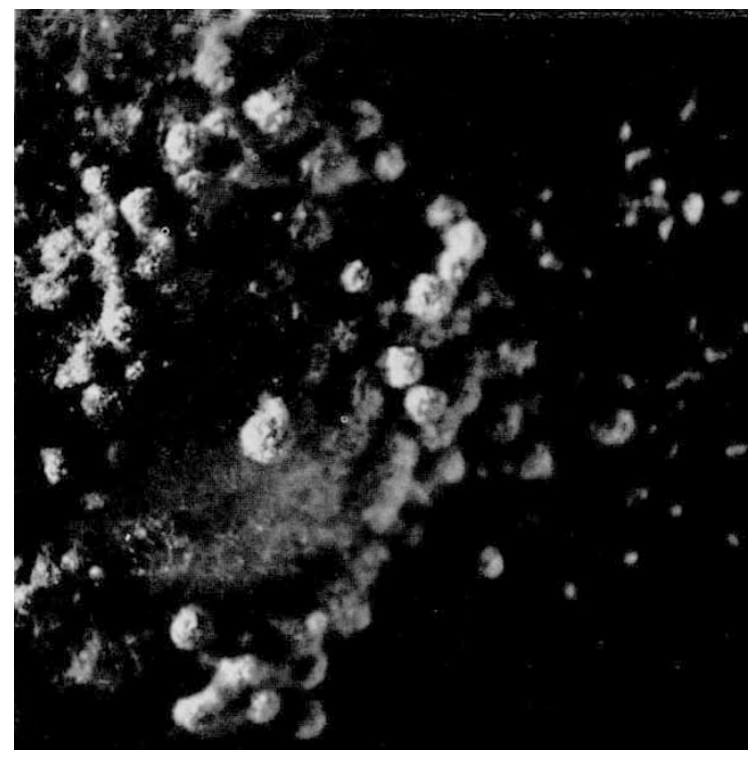

a.

The initiation of a $m$-normal sector by the outgrowth of hyphæ (bearing conidial heads) from the margin of a minute colony at a point where a clump of perithecia abut on it.

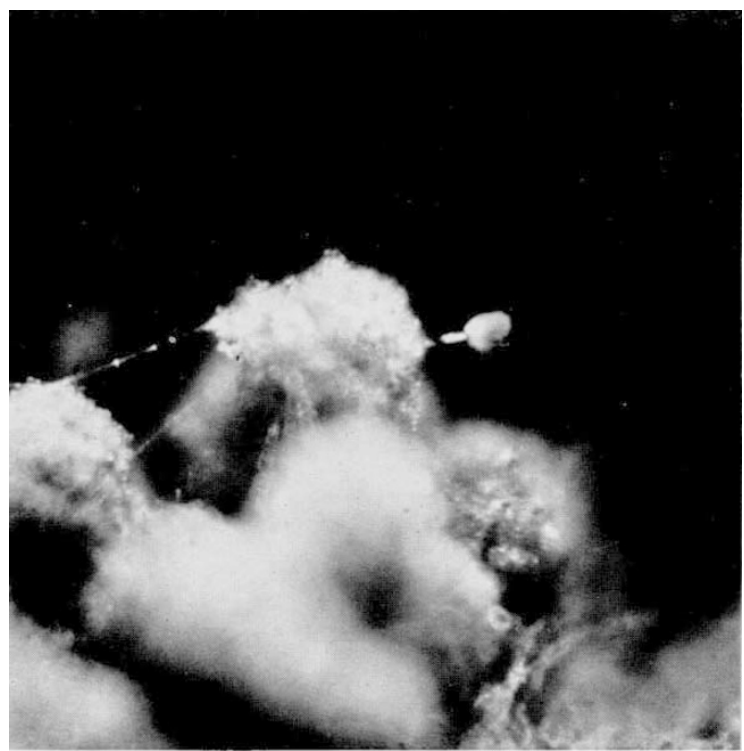

b.

An immature conidial head situated on, and derived from, the same hyphæ as the mass of Hülle cells surrounding a perithecium of the minute variant. 
5. The minute variant produces only $m$-normal sexual offspring. This indicates that only the $m$-normal homoplasmon is able to bring about the formation of ascospores.

6. It is therefore concluded that the cytoplasm, of the wildtype strain of $A$. nidulans, plays a critical role in the determination of the normal pattern of differentiation and development.

Acknowledgments.-This work was undertaken while the authors were in receipt of research studentships from the Department of Scientific and Industrial Research $\left(^{*}\right)$ and from the Agricultural Research Council ( $\dagger$ ). We are indebted to Prof. K. Mather, D.Sc., F.R.S., and Dr J. L. Jinks for advice and criticism.

\section{REFERENCES}

ARLETT, C. F. 1957. Induction of cytoplasmic mutations in Aspergillus nidulans. Nature, (Lond.), I79, I 250-I $25 \mathrm{I}$.

ARLETT, C. F., GRINDLE, M., AND JINKS, J. L. 1962. The "red" cytoplasmic variant of Aspergillus nidulans. Heredity, 17, 197-209.

EPHRUSSI, B. 1953. Nucleo-cytoplasmic relations in micro-organisms. Clarendon Press, Oxford.

EPHRUSSI, B., HOtTINGUER, H., AND CHIMÈNES, A. M. I949. Action de l'acriflavine sur les levures. I. La mutation "petite colonie". Ann. Inst. Pasteur, 76, $35 \mathrm{I}-367$.

EPHRUSSI, B., HOTTINGUER, H., AND ROMAN, H. 1955. Suppressiveness: a new factor in the genetic determination of the synthesis of respiratory enzymes in yeast. Proc. Nat. Acad. Sci., 4I, 1065-107I.

JiNks, J. L. I954. Somatic selection in fungi. Nature, (Lond.), I74, 409.

JINks, J. L. 1958. Cytoplasmic differentiation in fungi. Proc. Roy. Soc. B., I48, 3× 4-32I.

JINKs, J. L. I959. Lethal suppressive cytoplasms in aged clones of Aspergillus glaucus. 7. Gen. Microbiol., 21, 397-409.

JINKs, J. L. I963. Cytoplasmic inheritance in fungi. Methodology in basic genetics. Holden-Day, Inc., San Francisco.

pontecorvo, G. I953. The genetics of Asperigillus nidulans. Adv. in Genet., 5, $141-238$.

ROPER, J. A. 1958. Nucleo-cytoplasmic interactions in Aspergillus nidulans. Cold Spr. Hbr. Symp. Quant. Biol., 23, I4I-I 54 .

$\mathrm{SRB}, \mathrm{A} . \mathrm{M}$. 1958. Some consequences of nuclear-cytoplasmic recombinations among various Neurosporas. Cold Spr. Hbr. Symp. Quant. Biol., 23, $269-277$.

SRB, A. M. I963. Extrachromosomal factors in the genetic differentiation of Neurospora. Symp. Soc. Exptl. Biol., 17, I75-187.

yuILl, E. I950. The numbers of nuclei in conidia in Aspergilli. Trans. Brit. Mycol. Soc., 33, 324-33I. 